\begin{aligned} \hline NEW ATTACKS ON DOL EQUIVALENCE \\ PROBLEM \end{aligned}

\title{
EQUIVALENCE OF L-SYSTEMS
}

Mogens Nielsen

Department of Computer Science

University of Aarhus

Aarhus, Denmark

This paper summarizes some results concerning decidability of various kinds of equivalence problems for classes of L-systems - primarily DOL-systems. The reader is assumed to be famillar with some standard definitions and notations from the theory of L-systems.

For any finite alphabet, $\Sigma=\left\{\sigma_{1}, \sigma_{2}, \ldots, \sigma_{n}\right\}$, let $\pi_{\Sigma}$ denote the mapping that associates with each word from $\Sigma^{*}$ its corresponding Parikh-vector, i.e., for every word $x \in \Sigma^{*}, \pi_{\Sigma}(x)$ is the vector, in which the $i^{\prime}$ th component is the number of occurrences of $\sigma_{1}$ in $x$.

For any class of deterministic L-systems, you may consider equivalence with respect to

WL(WS) : the set (sequence) of words generated

PL (PS) : the set (sequence) of Parikh-vectors associated with the words generated

NL (NS) : the set (sequence) of lengths of the words generated Note that WL-, PL-, and NL-equivalence are also well-defined for nondeterministic L-systems.

The decidability of the corresponding six equivalence problems for DOL-systems is considered in $[70]$. The following result is proved $(|\Sigma|$ denotes the cardinality of $\Sigma$ ):

\section{Theorem 1}

For any two DOL-systems over some alphabet $\Sigma$, generating sequences of words $\left\{w_{1}\right\}$ and $\left\{v_{1}\right\}$ respectively:

$$
\begin{array}{ll}
\text { 1) } \forall i, 0 \leq i: & \pi_{\Sigma}\left(w_{1}\right)=\pi_{\Sigma}\left(v_{1}\right) \\
\text { iff } & \\
\text { 2) } \forall i, 0 \leq i \leq|\Sigma|: & \pi_{\Sigma}\left(w_{1}\right)=\pi_{\Sigma}\left(v_{1}\right)
\end{array}
$$

A direct consequence of Theorem 1 is

\section{Theorem 2}

PS-equivalence is decidable for DOL-systems.

Fur thermore, the following two theorems are proved in [70]: 


\section{Theorem 3}

PL-equivalence is decidable for DOL-systems.

\section{Theorem 4}

WL-equivalence is decidable for DOL-systems iff WS-equivalence is decidable for DOL-systems.

These theorems leave open one of the main open questions in the theory of L-systems, namely the decidability of WL- and WS-equivalence for DOL-systems. It is known that these equivalence problems are decidable for some subclasses of DOL, e.g., $([41]):$

\section{Theorem 5}

WL-equivalence is decidable for any class of unary L-systems (systems over a one-letter alphabet).

The following result is fairly easy to prove (a stronger version of the theorem has been proved by $P$. Johansen):

\section{Theorem 6}

WS-equivalence is decidable for locally catenative ([95]) DOL-systems.

Fur thermore, G. Rozenberg has proved:

\section{Theorem 7}

WS-equivalence is decidable for DOL-systems with polynomial growth ([75]).

On the other hand, WL- and WS-equivalence are also known to be undecidable for some classes of systems, that include DOL. The following two theorems are proved in $[80],[84]$, and $[99]$ :

\section{Theorem 8}

WL-equivalence is undecidable for POL-systems.

\section{Theorem 9}

WL-equivalence is undecidable for PDTOL-systems:

Furthermore, using an idea suggested by $P$. Vitanyi (originally to prove undecidability of NS-equivalence) you can prove: 


\section{Theorem 10}

All six equivalence problems considered in this paper are undecidable for D1L-systems.

It seems likely, however, that WS-and thereby WL-equivalence is decidable for DOL-systems. The following two results which are somewhat related to the problems are proved in $[70]$ :

\section{Theorem 11}

There exists an algorithm that will produce for any reduced ([70]) DOLsystem over an alphabet $\Sigma$, all (finitely many) systems over $\Sigma$, which are PL(PS-) equivalent to the given system.

\section{Theorem 12}

Let $S_{1}$ and $S_{2}$ be two WS-equivalent DOL-systems over an alphabet $\Sigma$, for which the first $|\Sigma|$ generated Parikh-vectors are linearly independent, then $S_{1}=S_{2}$.

(Note that the property "reduced" is decidable for OL-systems, but not for 1L-systems ([33])).

The following conjecture is suggested:

\section{Conjecture}

There exists a computable function $f$, mapping integers to integers, such that for any two DOL-systems over some alphabet $\Sigma$, generating sequences of words $\left\{w_{1}\right\}$ and $\left\{v_{1}\right\}$ :

$$
\text { 1) } \forall \mathrm{i}, 0 \leq \mathrm{i}: \quad \mathrm{w}_{1}=\mathrm{v}_{\mathrm{i}}
$$

2) $\forall i, \quad 0 \leq i \leq f(|\Sigma|): \quad w_{i}=v_{i}$

This conjecture implies, of course, the decidability of WS-equivalence for DOLsystems. Note the similarity between the conjecture and Theorem 1, which states that for sequences of Parikh-vectors generated, the conjecture is true with $f$ as the identity-function. That this is not the case for sequences of words generated, is seen from the following example.

\section{Example}

Consider the two DOL-systems, $S_{2}$ and $S_{z}$, over the alphabet $\Sigma=\left\{a_{1}, b_{1} \mid 1 \leq i \leq n\right\}$. 
$S_{1}$

axiom

productions $a_{1} b_{3}$

$a_{1} \rightarrow a_{2}$

$b_{1} \rightarrow b_{2}$

-

$\cdot$

$a_{n-1} \rightarrow a_{n}$

$b_{n-1} \rightarrow b_{n}$

$a_{n} \rightarrow a_{1} b_{1} b_{1}$

$b_{n} \rightarrow a_{1} a_{1} b_{1} b_{1} a_{1}$
$S_{2}$

$a_{1} b_{1}$

$a_{1} \rightarrow a_{2}$

$b_{1} \rightarrow b_{2}$

-

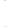

$a_{n-1} \rightarrow a_{n}$

$b_{n-1} \rightarrow b_{n}$

$a_{11} \rightarrow a_{1} b_{1} b_{1} a_{1} a_{1} b_{1} b_{1}$

$b_{n} \rightarrow a_{1}$

It is easy to verify that the sequences of words generated by these two systems coincide until the $3 n$ 'th generated word and no longer. This implies that if the above conjecture is true, then $f(i)>1 \frac{1}{z} \cdot i$ for every integer $i$.

Finally, concerning length-equivalence of DOL-systems [75]:

\section{Theorem 13}

NS-equivalence is decidable for DOL-systems.

Decidability of NL-equivalence is still an open question for DOL-systems. In [70] the following theorem was proved.

\section{Theorem 14}

NL-equivalence is decidable for PDOL-systems.

But the proof of Theorem 14 builds essentially on the propagating property of the systems, and furthermore, J. Karhumaki has shown that there exists a DOL-system for which the range of its growth-function is not the range of the growth-function of any PDOL-system. 